\title{
FRACTURE TOUGHNESS OF A LAMINATED COMPOSITE
}

\author{
Sharon Kao-Walter, Dept of Mechanical Engineering, Blekinge Institute of Technology, \\ Karlskrona, Sweden, e-mail:skw@bth.se \\ Per Ståhle, Div. of Solid Mechanics, Malmö University, Malmö, Sweden, \\ e-mail: pers@ts.mah.se \\ Rickard Hägglund, SCA Research AB, Sundsvall, Sweden, \\ e-mail: rickard.hagglund@research.sca.se
}

\begin{abstract}
The fracture toughness of a polymer-metal laminate composite is obtained by mechanical testing of a specimen containing a pre-crack. The laminate is a material used for packaging. It consists of a thin aluminium foil and a polymer coating. A centre cracked panel test geometry is used. Each of the layers forming the laminate is also tested separately. The result is compared with the measured fracture strength of the individual layers. It is observed that the load carrying capacity increases dramatically for the laminate. At the strain when peak load is reached for the laminate only aluminium is expected to carry any substantial load because of the low stiffness of the LDPE. However, the strength of the laminate is almost twice the strength of the aluminium foil. The reason seems to be that the aluminium forces the polymer to absorb large quantities of energy at small nominal strain. The toughness compares well with the accumulated toughness of all involved layers. Possible fracture of the interface between the layers is discussed.
\end{abstract}

\section{KEYWORDS}

Laminate, aluminium foil, polymer, crack, fracture toughness

\section{INTRODUCTION}

Liquid food packages are often made of packaging materials consisting of different material layers to fulfil several requirements of the package. It is very important to ensure that every layer maintains its function during the forming, filling and transportation processes. As an example here a liquid food packaging material is considered. This is a laminate consisting of LDPE (Low Density Polyethylene) and an aluminium foil (Al-foil). Several studies of different mechanical properties of these materials have been performed [1-5]. It was found in [2] that aluminium foil and LDPE laminated together provide significantly higher stress and strain at fracture as compared with the simplified analytical prediction. Related works can also be found in [6], [7], [8] where notched tensile strength, fracture toughness as well as fatigue resistance for fiber/aluminium composite laminate were studied.

The purpose of this work is to study the fracture toughness of a laminated material in relation to the adhesion between the layers. Load and extension were measured for a two-layer laminate specimen with a pre-crack as well as for the individual layers of the laminate. The same specimen geometry was used in all tests. For comparison, measurements were also done for the laminate without any adhesion between the layers. 


\section{MATERIAL}

Laminate in this work consists of Al-foil and Low Density Polyethylene (LDPE). Fully annealed AA1200 Al-foil and LDPE with the product name LD270 is used.

Load versus extension were measured for the following materials:

Case 1: Al-foil with the thickness $8.98 \mu \mathrm{m}$.

Case 2: LDPE with the thickness $27.30 \mu \mathrm{m}$.

Case 3: Al-foil / LDPE - Al-foil coated by LDPE and the total thickness is $36.28 \mu \mathrm{m}$.

Case 4: Al-foil // LDPE - Al-foil and LDPE joined together without adhesion between the layers and the total thickness is $36.28 \mu \mathrm{m}$.

Here "/" applies to two material layers bonded together and "//" applies to two layers put together without any adhesion in between.

For case 3, pieces of Al-foil were cut from a roll of fabricated material as shown in Fig.1(a). The laminate in case 3 was then prepared in a Haake film extruder with a $36 \mu \mathrm{m}$ Polyester (PET) as carrier (see Fig.1(b)). The foil was mounted on the PET carrier while LDPE was extruded and coated on Al-foil at a melting temperature of $278^{\circ} \mathrm{C}$. A nip with the pressure 202 bar was used to press the layers together. The laminated specimen for case 3 was than cut from roll including PET/Al-foil/LDPE as shown in Fig.1(c). After producing the material for case 3, LDPE was continuously extruded on the PET carrier under the same conditions but without the Al-foil. By peeling off the PET carrier, the LDPE produced here was used for making the specimen for case 2 and case 4 . And the specimen of case 1 is taken from the Al-foil roll of the same direction as the other cases.

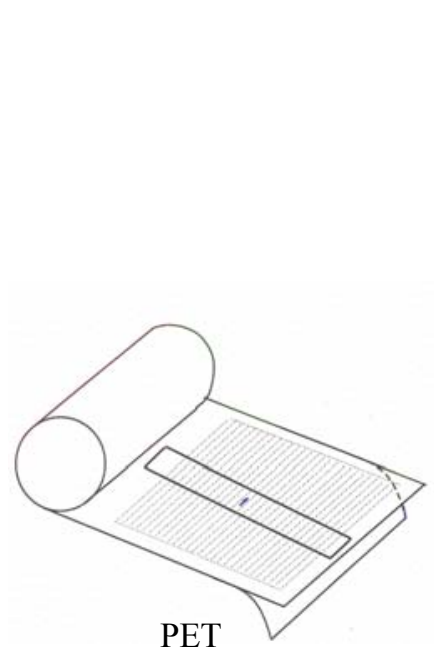

(c)

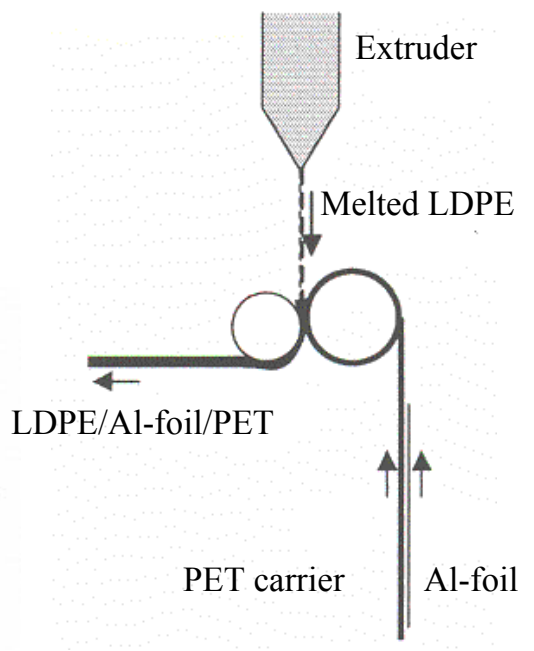

(b)

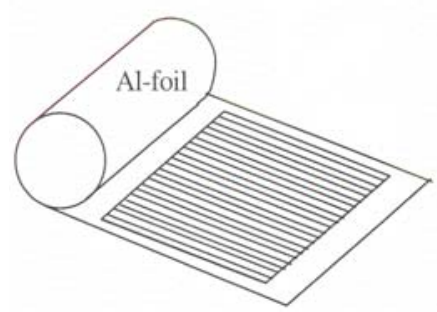

(a)

Fig.1. Schematic description of specimen preparing. 


\section{EXPERIMENTAL METHOD}

Centre cracked panels as shown in Fig.2(a) are used for evaluating the fracture toughness of the laminated composite and components of it. Pre-fabricated cracks are manually cut, using a razor blade, to a total slit length of $2 a=45 \mathrm{~mm}$. The width and gauge length of the specimens was $2 W=95 \mathrm{~mm}$ and $2 h=230 \mathrm{~mm}$, respectively.

A pair of wide clamps is utilised, see Fig.2(b). The tests are made in a MTS Universal Testing Machine. The upper clamp is attached to a $2.5 \mathrm{kN}$ load cell as well as a crosshead in the MTSmachine. Since the specimen is mounted vertically, the clamps are equipped with needles to facilitate a correct positioning. After the positioning of a sample the upper and lower clamps are closed and the pressure is applied by tightening four equally spaced quick-acting locking nuts along the front of each clamp, see Fig.2(b). Locking pins at the centre of the front jaws keep the clamps in an open position during mounting. The largest sample that can be accommodated is $420 \mathrm{~mm}$ wide. Specimens are tested by traversing the upper crosshead up to move the sample under increasing tension $u$ at a constant crosshead speed of $9.2 \mathrm{~mm} / \mathrm{min}$. The software TestWorks is used to control the load frame and also to record data. During testing both displacement between the crossheads and load is monitored and recorded. All tests are run until the entire cross section has fractured.

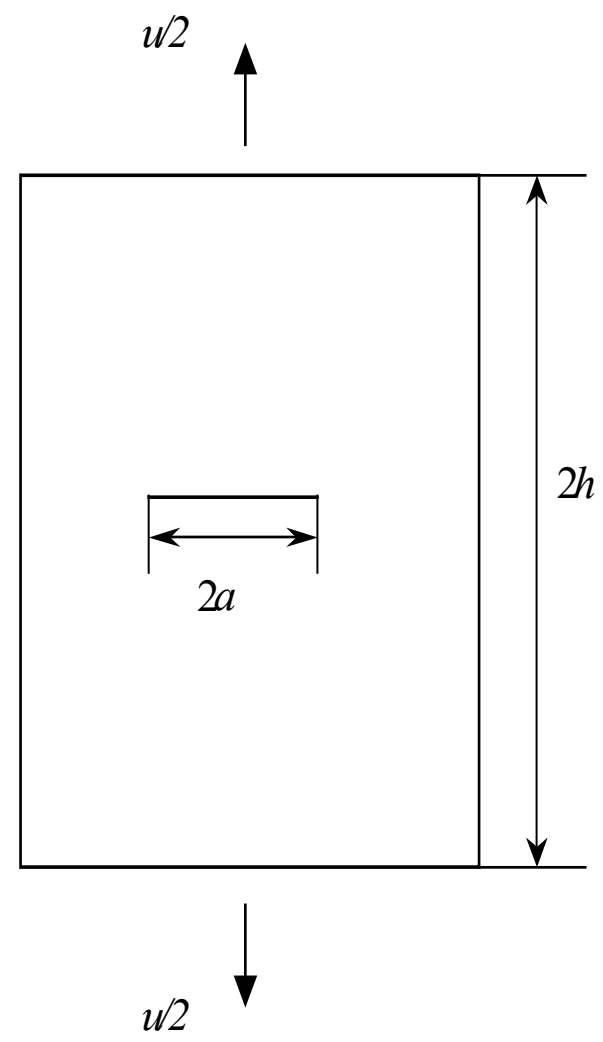

(a). Centre cracked panel.

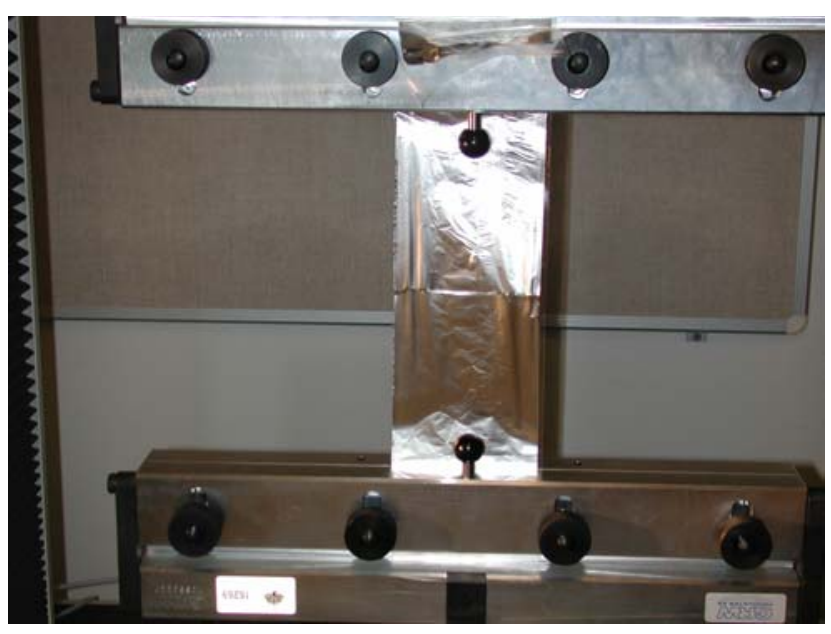

(b). Set-up for fracture mechanical testing of laminated composites. The specimen shown here is case 4. 


\section{ANALYTICAL APPROACH}

\section{ELASTIC BEHAVIOUR OF THE SPECIMEN}

The force elongating the specimen may be separated into that of the unbroken specimen, $P_{o}$, and the reduction, $P_{c}$, due to the presence of a crack. The former is calculated as follows:

$$
P_{o}=\frac{u E W t}{h}
$$

and the latter is obtained from the energy, $U_{c}$, released during the cutting of a crack [6]:

$$
U_{c}=\frac{t}{E} \int_{0}^{a} K_{\mathrm{I}}^{2} \mathrm{~d} a^{\prime}
$$

The stress intensity factor for the crack is given by

$$
K_{\mathrm{I}}=\frac{u E}{\sqrt{h\left(1-v^{2}\right)}} \phi(a / W)
$$

This gives the energy

$$
U_{c}=\frac{1}{2} u P_{c}=\frac{u^{2} E W t}{4 h\left(1-v^{2}\right)} \int_{0}^{a / W} \phi(\tau) \mathrm{d} \tau .
$$

Using $\phi$ given in [9] the integral of (4) is found to be 0.08 for $a / W=0.47$. With $W=47.5 \mathrm{~mm}$ and $h=115 \mathrm{~mm}(1),(3)$ and (4) give

$$
P=P_{o}-P_{c}=\frac{u E W t}{h}\left[1+\frac{1}{2\left(1-v^{2}\right)} \int_{0}^{a / W} \phi(\tau) d \tau\right]
$$

giving

$$
P=0.92 \frac{u E W t}{h}
$$

Thus, the stiffness of specimen decreases only $8 \%$ as compared with a corresponding unbroken specimen.

\section{CRACK TIP DRIVING FORCE G}

According to the theory of fracture mechanics, a crack tip driving force $G$ is defined as the rate of change in potential energy per crack area and per unit of length of crack front $[10,11]$. It is assumed that the critical driving force, $G_{c}$, of a crack in a single layer is constant and independent of whether the layer is bonded to other layers or not. Assuming that no delaminating occurs during the growth of a crack in the laminate and that energy is not dissipated in the material, the crack tip driving force for the laminate is the accumulated driving force for all layers, i.e. 


$$
G=\frac{G_{1} t_{1}+G_{2} t_{2}}{t_{1}+t_{2}}
$$

Here the assumption that $G=G_{C}$ as crack growth criterion is examined. This criterion is valid if the crack grows in an approximate steady state.

The load at onset of crack growth is usually regarded to define the fracture toughness. For materials with considerable toughening the incipient growth of the crack leads to increased fracture toughness. At small scale yielding the maximum toughness may be of interest but in the present analyses the yielding is considerable. There is a difficulty to define onset of crack growth since the observation is that the crack grows in the Al-foil until the crack traverses the entire specimen before there is any substantial crack growth in the LDPE layer.

\section{PEAK LOAD $F_{m}$ AND DISSIPATED ENERGY U}

For practical use the maximum load carry capacity may be limiting the reliability of the packaging structure. Therefore it is interesting to compare peak load both for each material separately and as a laminate. It is also interesting to examine the energy dissipated before breaking is reached. The dissipated energy, $U$, is found from the load displacement curve, $P(\delta)$ as follows

$$
U=\int_{0}^{\delta} P(\tau) \mathrm{d} \tau-\left.\frac{\mathrm{d} P}{\mathrm{~d} \delta}\right|_{\mathrm{d} \delta<0} \frac{\delta^{2}}{2}
$$

The second term represents the recovered elastic energy stored in the specimen. At the point marked with triangles (see curves from case 3 and case 4 in Fig.4) the crack has grown in the Al-foil and traversed the entire specimen whereas the crack in LDPE in most cases did not grow at all. The average strain at peak load is still in the elastic regime for the LDPE material. A first assumption would therefore be that the entire energy is due to the fracture of the aluminium and that the LDPE does not contribute to the fracture toughness. The motivation for the interest in only the dissipated part of the energy is that the elastic part may be recovered which means that accumulated energy, e.g. a shockwave may temporarily overcome the barrier that the elastic energy represents. If this results in crack advance the recovered energy is restored which may lead to rapid crack growth.

\section{RESULTS AND DISCUSSION}

\section{CALIBRATION OF LOAD CELL}

A load cell of $2.5 \mathrm{kN}$ has to be used because of the relatively large weight of the clamp. Prior to testing, the $2.5 \mathrm{kN}$ load cell was calibrated and accuracy was investigated. The result for the comparison with $100 \mathrm{~N}$ load cells is shown in Fig. 3. It can be seen that the deviation is around 


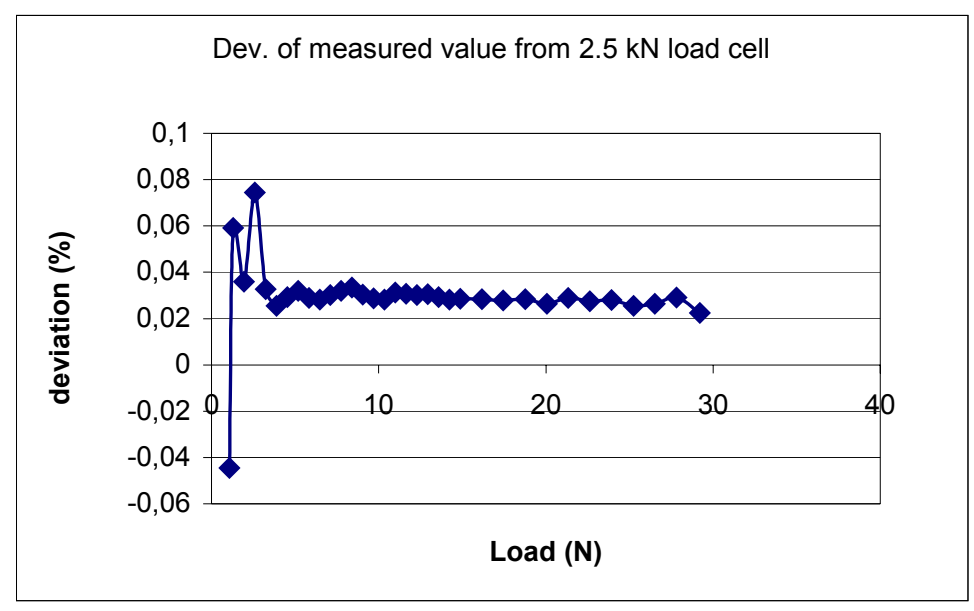

Fig.3. Difference in \% between measurements of a $100 \mathrm{~N}$ load cell and the $2.5 \mathrm{kN}$ load cell that is used in the experiment.

$0.03 \%$ for loads larger than $5 \mathrm{~N}$ and in the interval $1 \mathrm{~N}$ to $5 \mathrm{~N}$ the error is less than $0.08 \%$.

\section{MEASUREMENTS}

Measurements are made on a centre cracked panel as described above. The specimen is loaded via the clamps attached to the ends of the specimen.

The test speed is $9.2 \mathrm{~mm} / \mathrm{min}$ that gives a constant strain rate of $4 \%$ per min according to ASTM standard [12]. For each curve, five specimens are tested in room temperature. The averaged curves for the four materials are displayed in Fig.4. This figure shows the force versus extension diagram for the single layers together with the laminate.

For each case, the specimen was extended until the crack growth through the entire specimen and break to two peaces. For case 3 (Al-foil coated by a LDPE layer), it can be found that the maximum load value is the highest of all four cases. It was observed that the crack grows only in the Al-foil layer until the curve comes to the point that marked with a triangle where the crack in Al-foil traverses the entire specimen. Similar observations have been made for case 4 in the beginning of the curve. After passing the triangular point where crack in Al-foil traverses the entire specimen, the curve follows the curve of case 2 very well since there is only a single layer of LDPE remaining.

By experiment results maximum force $F_{m}$, dissipated energy before the onset of fracture $A_{m}$, total dissipated energy $A_{\text {tot }}$ as well as estimated stiffness $\mathrm{d} F / \mathrm{d} e$ in the elastic region are calculated and shown in Table 1. From these values and the diagrams in Fig.4, the results can be analyzed and discussed in the following. 


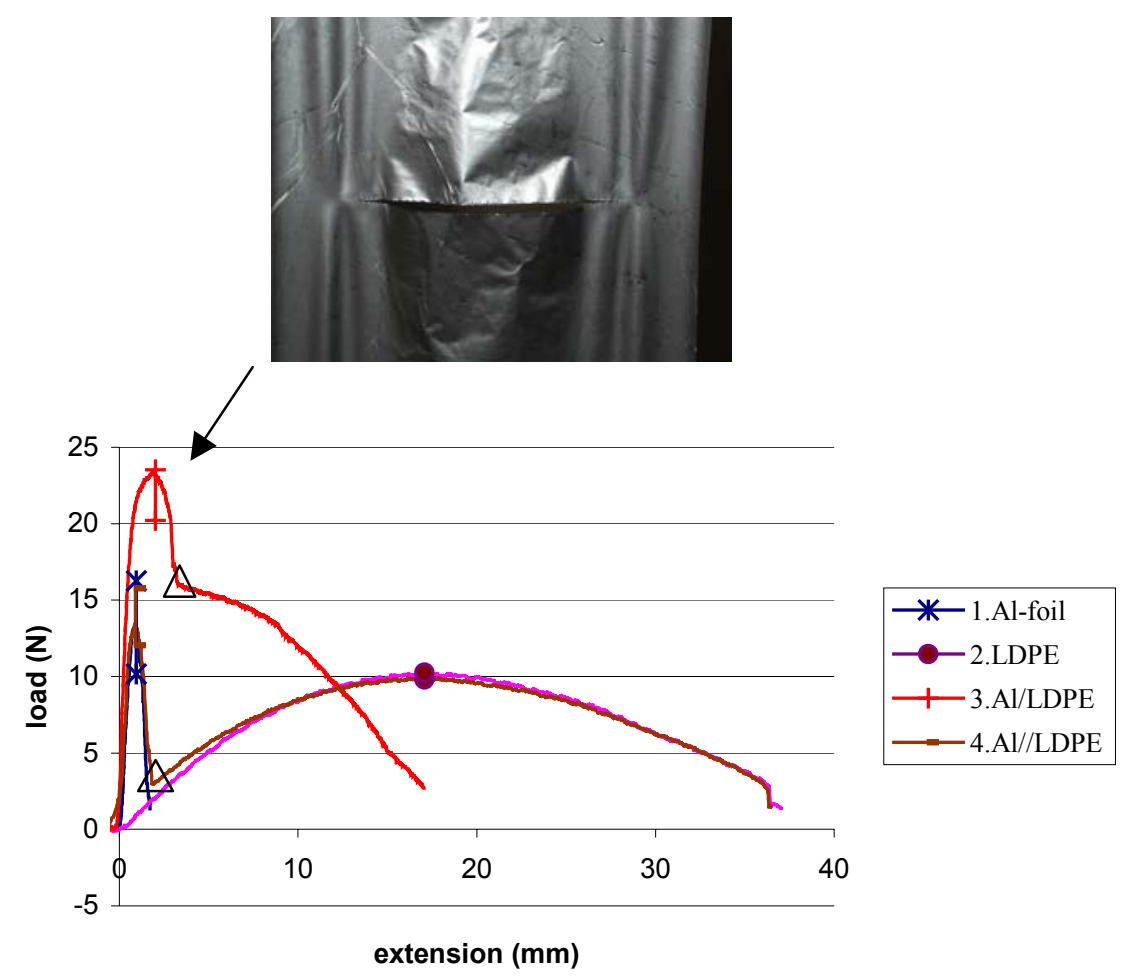

(a)

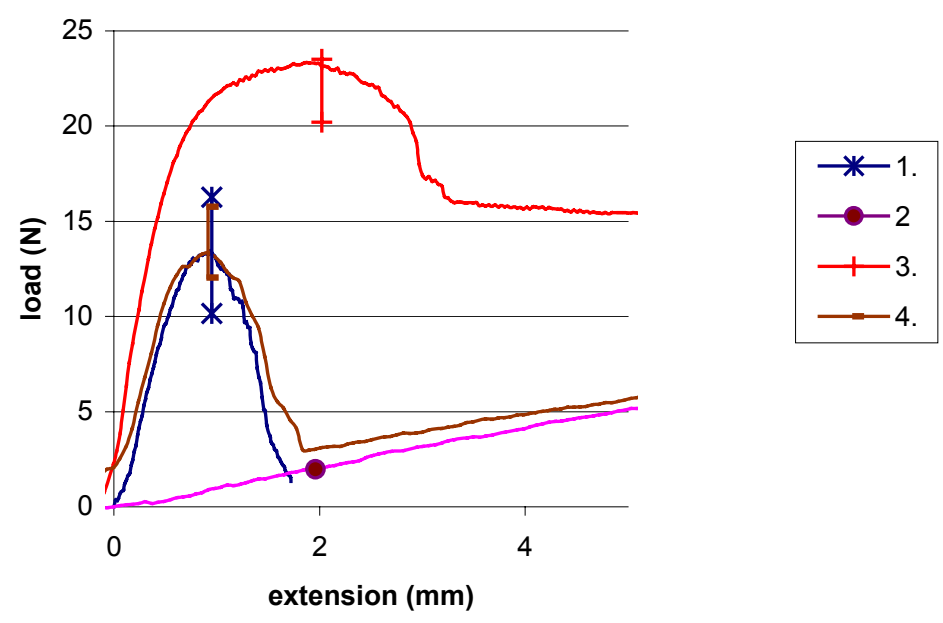

(b)

Fig. 4. Load versus extension for centre cracked panels. Diagram in (a) shows the results for one of the 5 tests from casel to case 4. The standard deviation of the peak load is shown on the curve for each case. Picture above was taken when the load for case 3 reaches the peak load. Diagram in (b) shows the detail result at extension from zero to $5 \mathrm{~mm}$.

The stiffness $d F / d e$ may be used together with (6) to predict the modulus of elasticity. The estimated modulus of elasticity is $9.3 \mathrm{GPa}$ for Al-foil and $92.9 \mathrm{MPa}$ for LDPE. The modulus of elasticity for aluminum is much smaller than expected (34 GPa in [2]). On the other hand, the modulus of elasticity for LDPE is rather close to the value from the earlier measured result (136 MPa in [2]). The measured displacements in the elastic region are therefore very uncertain and the stiffness results cannot be regarded as reliable. 
Table 1. Calculated values. $F_{m}$ is the maximum force, $A_{m}$ is the area under the curve from zero to maximum force. $A_{\text {tot }}$ is the total area under each curve of Fig.4. dF/de is the stiffness in elastic region Max and min refers to the largest and the smallest values obtained among the five tests.

\begin{tabular}{|c|c|c|c|c|}
\hline Cases & 1. Al-foil & 2. LDPE & 3. Al/LDPE & 4. Al//LDPE \\
\hline$F_{m}[N]$ & 12.9 & 10.0 & 22.5 & 14.2 \\
\hline$F_{m \max }$ & 16.3 & 10.3 & 23.5 & 15.8 \\
\hline$F_{m \text { min }}$ & 10.2 & 9.8 & 20.2 & 12.0 \\
\hline$A_{m}[\mathrm{~N} \mathrm{~mm}]$ & 6.3 & 114 & 32.7 & 8.1 \\
\hline$A_{\max }$ & 7.7 & 118 & 37.6 & 8.9 \\
\hline$A_{m \min }$ & 5.7 & 111 & 26.7 & 6.3 \\
\hline$A_{\text {tot }}[\mathrm{N} \mathrm{mm}]$ & 13.6 & 257 & 238 & 247 \\
\hline$A_{\text {tot } \max }$ & 15.5 & 264 & 331 & 273 \\
\hline$A_{\text {tot } \min }$ & 10.1 & 252 & 191 & 253 \\
\hline $\mathrm{d} F / \mathrm{d} e[N / \mathrm{mm}]$ & 31.1 & 0.97 & 25.3 & 25.6 \\
\hline$d F / d e_{\max }$ & 39.4 & 1.0 & 42.4 & 45.4 \\
\hline$d F / d e_{\text {min }}$ & 23.2 & 0.9 & 15.0 & 14.1 \\
\hline
\end{tabular}

However for case 4, the peak load was found to be the slightly larger than peak load for the Alfoil alone. The peak load 14.2 N may be compared with the peak load of aluminum $12.9 \mathrm{~N}$, the elongation at peak load of aluminum is $0.84 \mathrm{~mm}$ and the estimated stiffness in the elastic regime of LDPE is $0.97 \mathrm{~N} / \mathrm{mm}$. The latter gives an expected peak load of $12.9+0.84 \times 0.97=$ $13.7 \mathrm{~N}$ which is within $5 \%$ of the measured result $(14.2 \mathrm{~N})$.

For case 3, the laminate of LDPE and Al-foil joined together display a much higher peak load and larger extension at peak load. The value of this peak load of $22.5 \mathrm{~N}$ was found to be very close to the summation of peak load of Al-foil $(12.9 \mathrm{~N})$ and peak load of LDPE $(10 \mathrm{~N})$. During the test, small scale delamination between the layers was observed. The area under the load deflection curve, i.e., the energy required, is observed to be very large $(32.7 \mathrm{~N} \mathrm{~mm})$ compared with the energy required to break the Al-foil $(13.6 \mathrm{~N} \mathrm{~mm})$ and stored in the LDPE at the corresponding strain $(3.0 \mathrm{~N} \mathrm{~mm})$.

Direct visual inspection of Fig. 4 shows that, an astonishingly much larger amount of energy is consumed in the laminated material at moderate tension. The reason for this could be that the extension of LDPE requires multiple fracture of the Al-foil or delaminating. Multiple fracture of the Al-foil would consume a large amount of energy.

Examination of the total energy consumed during complete fracture of the specimens for case $1,2,3$ and 4 reveal that almost the same total energy is consumed at complete fracture irrespective of if the layers are bonded together or not. Here, energy required to break Al-foil is 13.6 $\mathrm{N} \mathrm{mm}$ and $257 \mathrm{~N} \mathrm{~mm}$ for the LDPE layer. This may be compared with the energy, $238 \mathrm{~N}$ $\mathrm{mm}$, required to break the laminate (case 3) and $247 \mathrm{~N} \mathrm{~mm}$, required to break both layers of material in case 4. It is believed that less energy is consumed during the fracture of the LDPE in the laminated case because the straining of the LDPE is concentrated to the thin gap that is defined by the broken Al-foil. The energy for onset the fracture in the Al-foil $\left(\mathrm{A}_{\mathrm{m}}^{\mathrm{Al}}=6.3 \mathrm{~N}\right.$ $\mathrm{mm}$ ) is certainly consumed already when the laminate has reached its peak load (compare to $\mathrm{A}_{\mathrm{m}}^{\mathrm{Al} / \mathrm{LDPE}}=32.7 \mathrm{~N} \mathrm{~mm}$ ). However, unexpectedly also the energy to break the laminate is almost entirely consumed at small straining of the specimen. The reason for this has to be sought in the mechanics of the fracture process region. 
The almost equal energies in case 4 and 3 rules out the hypothesis of presence of additional dissipative processes in the laminate as an explanation for the much higher toughness of the laminate. One observes also that more energy is consumed at small strains and, hence, less at larger strains. Further the peak load for the laminate is almost the same as the sum of the peak load for the Al-foil and the peak load for the LDPE layer. This suggests that the fracture processes distribute strain so that peak load occurs simultaneously in both materials. This is anticipated for a laminate with little delamination. The assumption is that both materials reach peak stress in a small process region in the vicinity of the crack tip.

\section{CONCLUSIONS}

An experimental investigation was performed on a laminate and separately on the individual components of the laminate. Peak load, energy dissipation at onset of fracture and at fast fracture were investigated. Fracture toughness cannot easily be defined because of very large scale yielding. Therefore an alternative toughness measurement was proposed.

The following observations were made:

- Onset of crack growth was observed to occur approximately at peak load. In the laminate fracture of LDPE occurred after completed failure of the Al-foil.

-Peak load for the laminate is almost the same as the sum of the peak load for the Al-foil and the peak load for the LDPE layer. This suggests that both materials reach peak stress in a small region in the vicinity of the crack tip.

-The total energy needed to complete fracture of the ligament in case 3 and case 4 are almost equal. The result rules out the hypothesis of significant additional dissipative processes in the laminate as an explanation for the much higher toughness of the laminate.

-The energy required before onset of fracture is unexpectedly large and around five times larger than for the separate Al-foil layer.

The last observation indicates a very important enhancement of the fracture toughness of the laminate that calls for further investigations. The finding is expected to be exploited in future composition of a broad range of laminates.

\section{ACKNOWLEDGEMENT}

This work was granted by Tetra Pak R\&D AB, Tetra Pak Carton Ambient AB, SCA Research $\mathrm{AB}$ and the Swedish KKs-Foundation. The authors would also like to thank Mr. K. Asplund and Mr. E.M. Mfoumou for assisting during the experimental work. 


\section{REFERENCES}

[1] Kao-Walter, S. and Ståhle, P. (2001) "In situ SEM study of fracture of an ultra thin Alfoil - modelling of the fracture process", SPIE 3rd ICEM proceeding.

[2] Kao-Walter, S., Dahlström, J. Karlsson,T. and Magnusson, A. (2002) "A study of relation between mechanical properties and adhesion level in a laminated packaging material", accepted by Mech. of Comp. Mat., Vol.39, Issue Nr.5-6, 2003.

[3 ] Lau, C. C. (1993) "A Fracture Mechanics Approach to the Adhesion of Packaging Laminates", Doc. Thesis, Imperial College of Science, UK.

[4] Tryding, J. (1996) "In Plane Fracture of Paper", Doc. Thesis, Division of Structural Mechanics, Lund Institute of Technology, Sweden.

[5] Kao-Walter, S. and Ståhle, P. (2001) "Mechanical and Fracture Properties of Thin Alfoil”, Research report, Blekinge Inst. of Tech., 2001:09.

[6] Macheret, J. and Bucci, R.J. (1993) "A crack Growth Resistance Curve Approach to Fiber/Metal Laminate Fracture Toughness Evaluation”, Eng. Frac. Mech. Vol.45, N0.6, pp.729-739.

[7] Gregory, M.A. and Roebroels, G.H.J.J. (1991) "Fiber/metal laminates: a solution to weight, strength and fatigue problems", $30^{\text {th }}$ Ann. Conf. of Metallurgical Society of CIM, Ottawa, Canada.

[8] Wells, J.K. and Beaumont, P.W.R. (1987) "The prediction of R-curves and notched tensile strength for composite laminates", J. Mater. Sci. 22, 1457-1468.

[9] Isida, M.(1971) "Effect of Width and Length on Stress Intensity Factors of Internally Cracked Plates Under Various Boundary Conditions", Int. J. Fract. Mech. Vol.7, p.301

[10] Anderson, T. (1995) "Fracture Mechanics: fundamentals and applications", 2 nd ed. CRC Press, USA.

[11] Irwin, G.R.(1948). "Fracture Dynamics" Fracture of Metals, American Society for metals, Cleveland.

[12] ASTM (1991) "Standard Test Methods for Tensile Properties of Thin Plastic Sheeting", D882-91. 